\title{
Exposure to Leadership WalkRounds in neonatal intensive care units is associated with a better patient safety culture and less caregiver burnout
}

\author{
J Bryan Sexton, ${ }^{1,2}$ Paul J Sharek, ${ }^{3,4,5}$ Eric J Thomas, ${ }^{6}$ Jeffrey B Gould, ${ }^{3,4,7}$ \\ Courtney C Nisbet, ${ }^{3,4}$ Amber B Amspoker, ${ }^{8,9}$ Mark A Kowalkowski, ${ }^{8,9}$ \\ René Schwendimann, ${ }^{2,10}$ Jochen Profit ${ }^{3,4,7}$
}

For numbered affiliations see end of article.

\section{Correspondence to} Dr Jochen Profit, Department of Pediatrics, Section of Neonatology, Stanford University, 1265 Welch Road, x115, Stanford, CA 94305, USA; profit@stanford.edu

At the time of this research, Dr Profit was a faculty member at Baylor College of Medicine, in the Department of Pediatrics and conducted his research at the Houston Veterans Affairs (VA) Health Services Research and Development Center of Excellence, Health Policy and Quality Program, Michael E DeBakey VA Medical Center, Houston, TX, USA.

Received 29 March 2013 Revised 21 April 2014 Accepted 22 April 2014 Published Online First 13 May 2014

\section{SLinked}

- http://dx.doi.org/10.1136/ bmjqs-2012-001706

- http://dx.doi.org/10.1136/ bmjqs-2014-003416

- http://dx.doi.org/10.1136/ bmjqs-2014-002831 CrossMark

To cite: Sexton JB, Sharek PJ, Thomas EJ, et al. BMJ Qual Saf 2014:23:814-822.

\begin{abstract}
Background Leadership WalkRounds (WR) are widely used in healthcare organisations to improve patient safety. The relationship between WR and caregiver assessments of patient safety culture, and healthcare worker burnout is unknown.

Methods This cross-sectional survey study evaluated the association between receiving feedback about actions taken as a result of WR and healthcare worker assessments of patient safety culture and burnout across 44 neonatal intensive care units (NICUs) actively participating in a structured delivery room management quality improvement initiative.
\end{abstract}

Results Of 3294 administered surveys, 2073 were returned for an overall response rate of $62.9 \%$. More WR feedback was associated with better safety culture results and lower burnout rates in the NICUs. Participation in WR and receiving feedback about WR were less common in NICUs than in a benchmarking comparison of adult clinical areas.

Conclusions WR are linked to patient safety and burnout. In NICUs, where they occurred more often, the workplace appears to be a better place to deliver and to receive care.

\section{INTRODUCTION}

In complex, fast-paced care settings such as the neonatal intensive care unit (NICU), patients are particularly vulnerable to medical errors. In the NICU, adverse events are common, frequently preventable and occur with great (10-fold) variation among NICUs. ${ }^{1}$ Executive or Leadership WalkRounds (WR) have been recommended as a tool to enhance patient safety in perinatal care. ${ }^{2}$

WR have enabled hospital leadership to sustain good relations with frontline caregivers, promote conversations to identify hazards and gather information to enhance decision making around patient safety. ${ }^{3}$ To date, however, relatively little research has been conducted on WR, and the best practices and aims of WR continue to evolve.

Experiences of clinicians and executives indicate that WR help educate leadership and frontline clinicians in patient safety concepts and lead to cultural changes such as increased transparency in discussions of adverse events and an improved rate of safety-based changes. ${ }^{4}$ WR have been associated with $64-80 \%$ increases in patient safety issues being resolved or having active progress reported. ${ }^{5}$

Ideally, WR create formal, although comfortable, venues for dialogue between leaders and frontline staff for identifying opportunities to improve care processes leading to better patient safety outcomes. ${ }^{6}$ Although the concept of WR is popular and widely used in North American hospitals, ${ }^{7-10}$ the link between WR and specific patient safety attitudes of clinical staff as well as clinical patient outcomes is not well established. One barrier to rigorous examination of the effects of WR is that their format varies widely depending on the institutions in which they are conducted and the leaders conducting them. WR frequencies range from weekly across the hospital ${ }^{11}$ to 
monthly visits in the same hospital units or clinical areas, to random visits to random units once a month. ${ }^{5} 12$ They may be conducted as formal sessions with auditorium presentations, discussions with staff over snacks or tea ('Safe-tea Time') or as informal hallway conversations. Documentation of WR also varies considerably, with some institutions capturing detailed written minutes of the WR discussions and others insisting that no names and details be written down. ${ }^{13} 14$

In addition, it is often difficult to measure the frequency of WR use in a particular clinical area. For example, there are consistent discrepancies between whether nurse managers reports that WR occur and what their staff report about personally attending a WR session. ${ }^{15}$ It is not uncommon for enthusiastic leaders to visit a unit and speak with the same two or three staff members each month, which is great for those staff members, but does not disseminate the concept of WR evenly. A recent study of WR exposure suggested a method for assessing WR exposure that measures the extent to which staff report having personally received feedback about actions taken to reduce patient safety risks as a result of WR in their clinical area. ${ }^{15}$

This may be a helpful indicator of WR exposure because it implies several critical factors: (1) WR are occurring on an ongoing basis in a specific clinical area; (2) safety issues are being surfaced and resolved through the WR sessions; and (3) patient safety risks reduced through WR are being fed back to staff in the clinical area, closing the loop and demonstrating the efficacy of the WR sessions.

WR are suggested to improve patient safety via engagement of frontline workers in creating a culture of safety, but to date, little is known about the relation between WR, safety culture and workforce engagement. We explored the association of WR feedback with multiple safety culture and workforce engagement domains, including caregiver burnout. Given that a third of physicians ${ }^{16}$ and nurses ${ }^{17}$ meet the criteria for burnout, another potential benefit of finding and fixing safety defects is to enhance patient safety engagement among caregivers. This increased engagement, without substantially increasing effort, has potential to reduce the alarmingly high levels of burnout. ${ }^{18}$ Reducing burnout is important because burnout is associated with a variety of healthcare worker and patient outcomes. ${ }^{19}$ Few studies have linked exposure to WR with changes in safety culture, and to our knowledge, none have linked them to caregiver burnout.

The aims of this study were to evaluate the association between WR feedback, patient safety culture and caregiver burnout. We hypothesised that the WR feedback was positively associated with safety culture domains and negatively associated with caregiver burnout.

\section{METHODS}

\section{Overview}

This cross-sectional survey study was performed among a convenience sample of NICUs participating in a Delivery Room Management Quality Improvement Collaborative organised by the California Perinatal Quality Care Collaborative (CPQCC). ${ }^{20}$ For the current study of NICUs, we assembled a survey to investigate safety culture and workforce engagement using existing validated metrics from multiple instruments (detailed below). In addition, we provide a broader perspective on WR exposure by comparing the NICU setting with other hospital units using a survey of 706 units in 49 hospitals, ${ }^{15}$ in which we have previously examined indices of exposure to WR as reported by healthcare workers. That study setting consisted of a mix of clinical areas (eg, medical-surgical units, pharmacy, intensive care units, radiology) and included caregivers such as nurses, physicians, technicians, support staff and others directly or indirectly involved in patient care.

\section{Selection of NICUs}

We offered to analyse and feed back a survey of safety culture and workforce engagement to all 61 NICUs who participated in the improvement initiative, 44 of which accepted. The survey was administered at the onset of the improvement initiative (between June and September 2011). Of the 44 NICUs, 10 (22.7\%) were designated as regional NICUs, $28(63.6 \%)$ as community NICUs and $6(13.6 \%)$ as intermediate NICUs as defined by the California Department of Healthcare Services. These designations are roughly equivalent with designations by the American Academy of Pediatrics as levels 4, 3 and 2, respectively. ${ }^{21}$

Staff with 0.5 full time equivalent (FTE) or more in the NICU for at least the four consecutive weeks prior to survey administration were invited to participate. Paper-based surveys were administered during regularly scheduled departmental and staff meetings, together with a pencil and return sealable envelope to maintain confidentiality. Individuals not captured in pre-existing meetings were hand delivered a survey, pencil and return envelope. This administration technique has generated high response rates. ${ }^{22} 23$ Administration of the survey was executed by CPQCC, and a de-identified data set was transmitted to Dr Profit for analysis.

\section{Measures}

Measures relevant for this paper came from a survey of safety culture and workforce engagement. These included select items on participation in Leadership WR, ${ }^{12} 24$ and scales from the Safety Attitudes Questionnaire (SAQ), ${ }^{23}$ the Hospital Survey on Patient Safety Culture (HSOPSC) ${ }^{25}$ and the Maslach Burnout Inventory. ${ }^{26}$ 


\section{Leadership WR exposure variables}

In this survey, WR exposure was assessed in three ways: (1) do they occur here, (2) have you directly participated and (3) have you received feedback about actions taken to reduce risks. All three of these frames yielded similar patterns of results, but in particular it was the extent to which participants had received feedback about actions taken to reduce risks that 'revealed significant differences between units with relatively high WR exposure compared with those units with relatively low WR exposure'. ${ }^{15}$

Anecdotal WR issues include identification of lack of clarity of chain of command, challenges obtaining equipment and supplies in a timely fashion, and lack of access to critical secured areas for some delivery room personnel. The nature of leadership WR is to discuss patient safety issues and concerns at the unit level and includes appropriate follow-up actions using the leader's position to make things happen at hierarchical levels above individual units. Accordingly, providing feedback to the involved unit caregivers about actions taken as a result of these WRs is essential to keep momentum and build trust in management's ability to solve patient safety problems. For instance, unit managers and safety officers track planned measures at the unit or departmental level following WR for updates and communicate this follow-up information back to caregivers and senior leadership aiming to supply accurate feedback to ensure completion of improvement tasks. ${ }^{3}$

We used the indices of WR exposure in the current study by incorporating three questions from a version of the SAQ modified to include specific WR-related items: (1) "Does this NICU use Patient Safety Leadership WalkRounds to discuss with senior leaders any issues that could harm patients or undermine the safe delivery of care?: (yes; no; not Sure)" and (2) "How often did you participate? $(0 ; 1 ; 2 ; 3-4 ; 5-7 ; 8$ times or more; not sure)" and (3) "Did you receive feedback about patient safety risks that were reduced as a result of WalkRounds? (yes; no; not sure)" The last item was our self-reported caregiver assessment of the extent to which patient safety issues were elicited and acted upon to reduce risk, and then fed back to caregivers. These items did not contain a specific reference timeframe.

\section{Patient safety culture}

Of the several safety culture survey instruments in the literature, the SAQ and the HSOPSC are widely used and have good psychometric properties. ${ }^{23} 27$ The SAQ is associated with clinical outcomes ${ }^{28-31}$ and contains 30 items that load on six domains: teamwork climate, safety climate, job satisfaction, perceptions of management, stress recognition and working conditions with response scales ranging from 1 (disagree strongly) to 5 (agree strongly). In this paper, we present the results of the safety climate and teamwork climate domains. The SAQ also captures respondent characteristics, including job position, years in specialty, primary work area (paediatric, adult or both), gender and predominant work shift. Job positions included attending physicians (MDs), fellow MDs, neonatal nurse practitioners (NNPs), registered nurse (RNs), respiratory care practitioners (RTs) and others.

The HSOPSC ${ }^{27}$ is composed of 42 items that are separated into scales to measure 12 domains of safety culture. For the current study, part of a larger study of safety culture and organisational determinants of quality in NICUs, we included four HSOPSC domains: overall perceptions of safety, teamwork within units, communication openness and error feedback and communication. An example item from error feedback and communication is, "We are given feedback about changes put into place based on event reports". For consistency, and to save room on the paper-based survey administration, the HSOPSC items were scaled using the standard SAQ Likert options ranging from 1 (disagree strongly) to 5 (agree strongly). This differed slightly from the HSOPSC standard, which uses several Likert options (eg, never/ rarely/sometimes/most of the time/always), but did not change the phrasing of the items. We report internal consistency reliability of all scales below. Using the standard and published technique for the SAQ and HSOPSC, the scale scores were calculated as the per cent of respondents within a NICU that had a mean equivalent across all items of 'agree slightly or agree strongly'. 3233

\section{Burnout}

To assess burnout, we used four items from the emotional exhaustion subscale of the Maslach Burnout Inventory, ${ }^{26}$ a technique that we have shown to be reliable and valid in previous work. ${ }^{32}$ The four emotional exhaustion items were: "I feel burned out from my work"; "I feel frustrated by my job"; "I feel I am working too hard on my job" and "I feel fatigued when I get up in the morning and have to face another day on the job." The response scale ranged from 1 (disagree strongly) to 5 (agree strongly). Using a published technique, the burnout scores were calculated as the per cent of respondents within a clinical area that had a mean equivalent across all four items of 'neutral or higher'. 32

\section{Statistical analysis}

We used descriptive analyses such as frequencies, percentages, means $( \pm S D)$ and graphs to describe demographics, exposure to leadership WR feedback, safety culture scales and caregiver burnout.

Scale scores were calculated for individual NICUs by taking the average of the scaled items, and then calculating the percentage of respondents within a NICU who reported positively (ie, proportion of 
those who agreed slightly or strongly). ${ }^{33}$ Scale reliability was assessed via Cronbach $\alpha$.

Leadership WR exposure was aggregated at the unit level by calculating the percentage of respondents within a unit who reported using, participating in and receiving feedback about WR. Any number of participations ( 1 through 8 or more) was categorised as a 'yes'.

Burnout was computed by taking the mean of the four items, transforming them to a $0-100$ point scale and calculating for each individual whether they had an average score of greater than or equal to 50 (ie, on average rating items as neutral, agree slightly and agree strongly). Then we determined the percentage of individuals within each NICU who had an average score of greater than or equal to 50 . We call this the 'percentage reporting burnout'. We have used a similar approach with safety culture assessments and found the metric to be meaningful to providers. ${ }^{13} 2334$

We used independent samples $t$ tests to examine whether mean differences in scale scores were associated with exposure to WR feedback, and also to test for differences between NICUs and adult clinical areas regarding the WR exposure variables.

All analyses were performed using IBM SPSS Statistics (V.20; IBM Inc., Armonk, New York, USA).

\section{RESULTS}

\section{Sample}

Forty-four NICUs participated in this study. Of 3294 administered surveys, 2073 were returned for an overall response rate of $62.9 \%$. Response rates within NICUs ranged from $21.7 \%$ to $100 \%$ with an average of $69.7 \%(\mathrm{SD}=19.8 \%)$, and the number of respondents per NICU was $\bar{x}=47.1$ ( $\mathrm{SD}=24.7)$, ranging from 10 to 144 respondents. Table 1 lists respondent characteristics. Of the 1962 respondents who indicated their position and length of experience, 1175 (59.9\%) reported at least 11 years in their specialty. Only 47 $(2.4 \%)$ reported less than 1 year in their specialty. Attending MDs were predominantly male (58.8\%). All other positions were predominantly female (fellow MDs 61.3\%, RNs 94.1\%, NNPs 100\%, RTs 53.5\%).

\section{Leadership WR exposure}

Results are shown in figure 1. WR utilisation in NICUs ranged from $5.3 \%$ to $63.3 \%$ and was on average not significantly different from adult clinical areas (mean\% (SD), 30.1 (13.9) vs 30.4 (20.0), $\mathrm{p}=0.90)$. WR participation: all 44 NICUs had respondents who reported that they themselves had participated at least once, and this ranged from $3.1 \%$ to $44.0 \%$. WR participation was less common in NICUs than in adult clinical areas $(20.9(10.5)$ vs $27.6(20.2)$, $\mathrm{p}<0.001)$. There were also large proportions of respondents who reported that they were not sure whether WR were occurring (49.1\%), and 35.8\% were not sure whether they had even participated in WR. WR feedback ranged from $3.3 \%$ to $43.5 \%$ in the

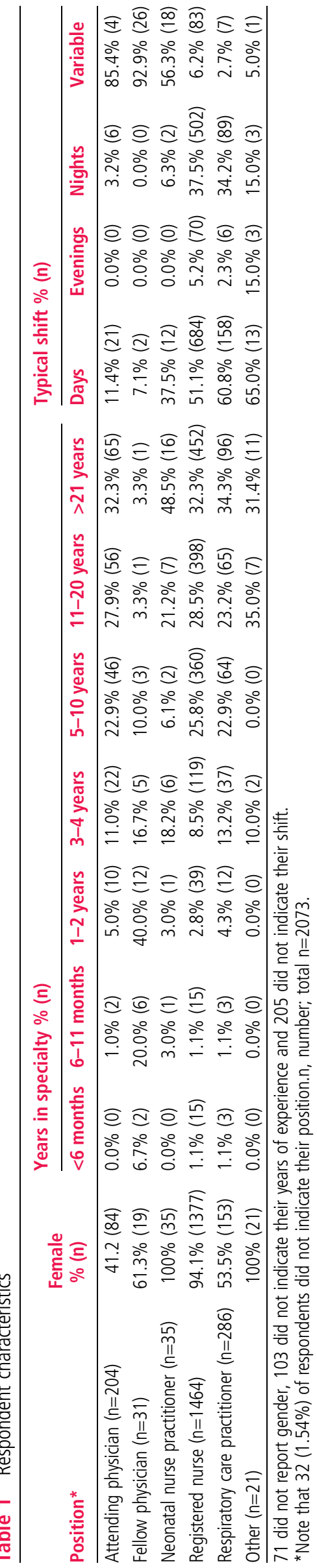



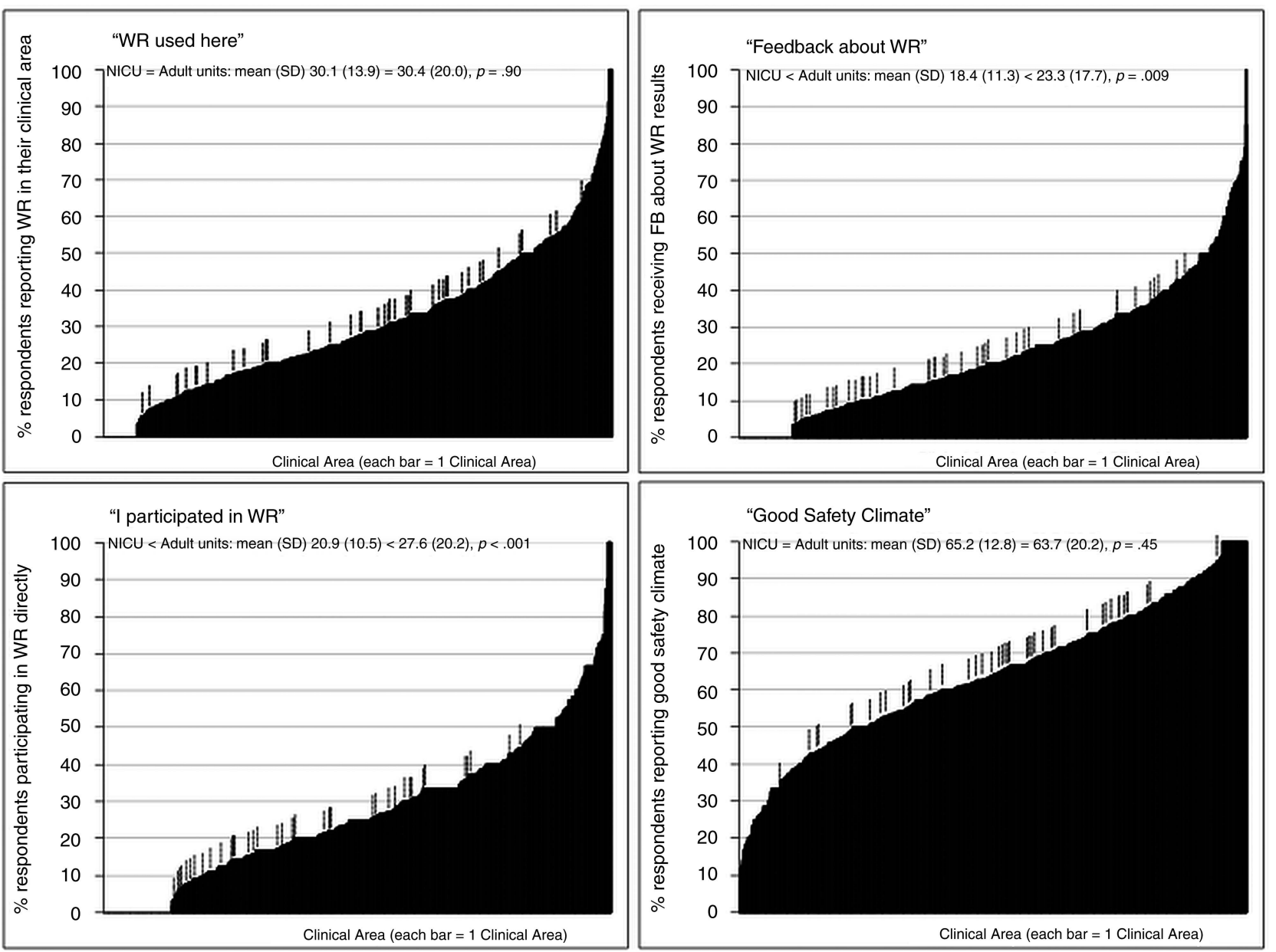

Figure 1 Three panels of WalkRounds exposure and one for safety climate. Each bar=1 clinical area, hash marks indicate a neonatal intensive care unit (NICU). There are 44 NICUs and 706 adult clinical areas from: Schwendimann R, Milne J, Frush K, Ausserhofer D, Frankel A, and Sexton JB. American Journal Of Medical Quality: 28 January 2013. The mean (SD) number of respondents per adult clinical area was $27.0,46 \%$ of which were RNs (by comparison, the NICU mean was $47.1,71 \%$ of which were RNs). RN, registered nurse.

NICU and was significantly lower than adult clinical areas $(18.4(11.3)$ vs $23.3(17.7), \mathrm{p}=0.009)$.

\section{Scale scores from SAQ, HSOPSC and the Maslach Burnout Inventory}

Consistent with prior psychometric work, we found good internal reliability for the safety culture scales from the SAQ (teamwork climate $\alpha=0.80$, safety climate $\alpha=0.80$, from HSOPSC: teamwork within units $\alpha=0.86$, communication openness $\alpha=0.70$, feedback and communication about error $\alpha=0.80$, overall perceptions of safety $\alpha=0.74$ and burnout $\alpha=0.85$ ). Our previously published survey of 706 adult units in 49 hospitals ${ }^{15}$ served as benchmarking data for the current study as it included safety climate and the three WR exposure variables. Figure 1 shows the proportion of respondents who reported good safety climate in each NICU ranged from $33.3 \%$ to $95.0 \%(\bar{x}=65.2, \mathrm{SD}=12.8)$, and overall safety climate across NICUs was not significantly different from safety climate across adult clinical areas. The percentages of respondents in each NICU reporting burnout ranged from $7.5 \%$ to $54.4 \%$ (on average, $25.9 \%, \mathrm{SD}=10.8$ ).

Scale scores by quartiles of exposure to WR feedback Comparison of the first and fourth WR feedback quartiles yielded significant differences in the SAQ domains of safety climate and teamwork climate (see figure 2). Comparison of the first and fourth WR feedback quartiles yielded significant differences in two of the four HSOPSC domains, overall perceptions of safety and feedback and communication about error (see figure 2). Comparison of the first and fourth WR feedback quartiles and burnout trended $(p=0.07)$, but did not reach statistical significance.

\section{DISCUSSION}

We found a consistent pattern of results, whereby WR feedback was associated with better safety culture domains and lower burnout. This retrospective multicentre study also compared exposure with WR 


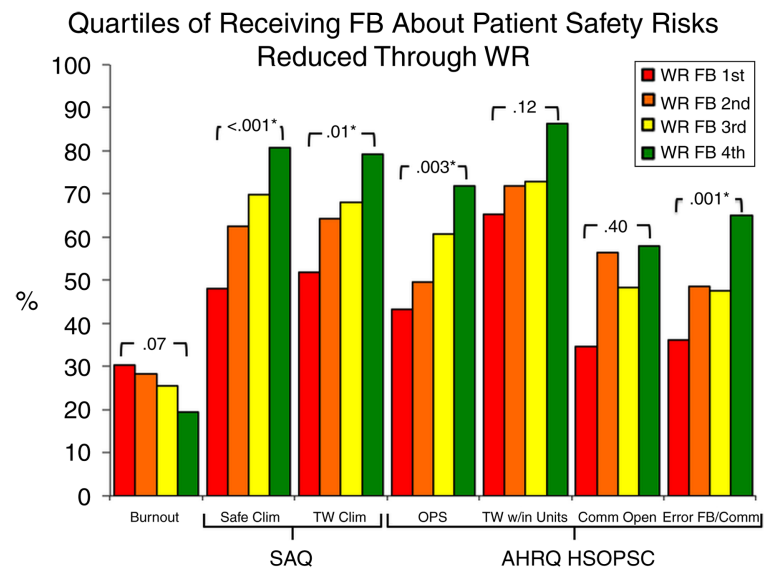

Figure 2 Burnout and safety culture domains displayed by WR feedback quartiles. Comparison of the first and fourth WR feedback quartiles with domains of safety culture and burnout. SAQ, Safety Attitudes Questionnaire; AHRQ HSOPSC, Agency for Healthcare Research and Quality Hospital Survey on Patient Safety Culture; Safe Clim, safety climate; TW Clim, teamwork climate; OPS, overall perceptions of safety; TW w/in units, teamwork within units; Comm Open, communication openness; Error FB/Comm, error feedback and communication; WR, WalkRounds.

feedback in 44 NICUs to 706 adult clinical areas, highlighting that while one out of three respondents report that WR are used in their NICUs and adult clinical areas, rates of direct WR participation and exposure to WR feedback were significantly lower in NICUs.

Our results expand on previous assessments of WR efficacy by adding the domain of burnout and by using safety culture domains from the two most widely used safety culture surveys, for a total of seven scale scores in the current study. More WR feedback was associated with significantly better safety climate, teamwork climate, overall perceptions of safety and feedback and communication about error; and a trend towards better teamwork within units and lower burnout. The results for communication openness were not significant and did not follow the overall pattern as closely. The results linking WR feedback to safety culture were strongest for safety climate, feedback and communication about error, and overall perceptions of safety, which is consistent with the conceptualisation of WR as an opportunity to find, discuss and fix patient safety problems. Using the WR feedback method may help researchers explore the efficacy of various frequencies, formats, durations and even overall objectives for using WR. What we assessed with WR frequency assumes that WR are happening, that issues are surfaced and discussed, that solutions are attempted and that the outcomes of those solutions are being fed back to frontline healthcare workers.

Our finding of an association between WR feedback and decreased burnout is important given its high prevalence in the medical field, its association with poor health outcomes for caregivers ${ }^{35-38}$ and patients, ${ }^{23} 39-42$ and the dearth of effective interventions to reduce healthcare worker burnout. ${ }^{43}$ Our finding gives rise to an important hypothesis that needs to be tested prospectively. This relation may signal that direct caregiver engagement in patient safety activities at a pace and intensity that is manageable may validate frontline expertise and provide meaning to daily work in a way that reduces burnout. This is important because the ability to reduce burnout is associated with improved teamwork climate $^{23}$ and reduced rates of medical errors ${ }^{39} 4042$ and suboptimal care. ${ }^{41}$

We double-checked the psychometric soundness of the scales used in the current study. The SAQ and Burnout domains exhibited good internal consistency, from 0.8 to 0.85 , consistent with previous work. However, the HSOPSC domains exhibited better psychometric properties than previously published work. ${ }^{27}$ Blegen et al's HSOPSC psychometric work found Cronbach $\alpha$ for overall perceptions of safety (0.53), teamwork within units (0.77), communication openness (0.64) and error feedback and communication (0.82). In the current study, we modified the response options to keep it consistent with the rest of the survey and found Cronbach $\alpha$ of $0.74,0.86,0.70$ and 0.80 , respectively. These improvements in the internal consistency of HSOPSC scales may be a statistical artefact, or may reflect our conversion of response options, or something else, but it suggests that further minimal refinement to HSOPSC may yield enhanced psychometrics of this widely used instrument.

The limitations of this study need to be viewed in light of its design. Cross-sectional surveys allow observations and associations to be made, whereas causal relationships between WR feedback and improvements in safety culture domains cannot be established. In addition, there was substantial variability between NICUs with regard to the item "Does your clinical area use Patient Safety Leadership WR to discuss with senior leaders any issues that could harm patients or undermine the safe delivery of care?”, which tempers the conclusiveness of our findings. We added 706 adult clinical areas to the analyses to explore the question of WR exposure and included additional analyses between these previously published adult clinical areas and our 44 NICUs. Although averaging across these two groups may limit the granularity of the analysis, WR appeared to be used evenly across adult and NICU settings, but in NICUs there was less direct participation in WR and less feedback about actions taken.

This difference might be explained by a number of factors. Perhaps it is easier to get WR participation and provide feedback in adult clinical areas based on the number of healthcare workers involved. There 
were 27 respondents in the average adult clinical area, but there were 47 respondents in the average NICU. In addition, the percentage of nurses responding to the survey was higher in the NICUs than in the adult units $(71 \%$ vs $46 \%)$, and their experiences with regard WR participation and WR feedback may be different.

Another limitation that may temper our findings is the variable response rates between the participating NICUs. It is possible that these may have introduced bias, the direction of which is impossible to determine. In addition, alternate ways to identify and act on staff concerns might affect our findings. Nevertheless, the inclusion of 706 additional units and 5 additional safety culture domains provided additional support for the consistency of our results, the patterns and our conclusions.

In addition, feedback about patient safety risks that were reduced as a result of WR was reported by 5.3$63.3 \%$ of respondents within a NICU. This highlights the difficulty for users to effectively raise awareness about this intervention among the staff, especially those that are not routinely exposed to them (eg, night shift, non-nursing providers, etc.). It also raises concern whether WR feedback, given this low penetration, is responsible for the effects on safety culture domains and burnout. It is possible that effects from unobserved confounding variables, such as significant disruptions of the work environment, such as leadership changes, may have influenced our findings. On the other hand, one might also argue that we found significant associations despite a relatively low penetration of the intervention, highlighting the need for more effective strategies to raise staff awareness of WR in order to reach its theoretical potential.

Exploring the specific patient safety risk reduction strategies or the format and content of WR was beyond the scope of the current study. Nevertheless, the results suggest that higher WR frequency was associated with larger proportions of respondents reporting that they thought one or more patient safety risks were reduced as a result. In addition, our focus on rates of WR exposure is a methodological option that others may choose to explore as it allows for systematic comparisons between units, over time, regardless of the type of unit, background of the senior leader, length of the WR, location of the WR and dynamics between the caregivers and the senior leaders.

\section{CONCLUSION}

We tested a new method for evaluating WR that consisted of measuring the extent to which staff report having personally received feedback about actions taken to reduce patient safety risks as a result of WR in their clinical area. Our findings indicate that more WR feedback was associated with better safety culture on both the SAQ and the HSOPSC instruments. WR feedback also appears to be associated with healthcare worker burnout, which suggests that WR may not be used to its full potential when feedback about actions taken as a result of WR is not provided to provide closure to the original WR visit. While WR appear to occur with the same frequency across NICUs and clinical areas, NICU respondents do not participate directly in WR nor do they receive WR feedback as often as participants in adult clinical areas.

Author affiliations

${ }^{1}$ Department of Psychiatry, Duke University School of

Medicine, Duke University Health System, Durham, North

Carolina, USA

${ }^{2}$ Patient Safety Training and Research Center, Duke University

Health System, Durham, North Carolina, USA

${ }^{3}$ Division of General Pediatrics, Department of Pediatrics,

Stanford University, Palo Alto, California, USA

${ }^{4}$ California Perinatal Quality Care Collaborative, Palo Alto,

California, USA

${ }^{5}$ Center for Quality and Clinical Effectiveness, Lucile Packard

Cahildren's Hospital, Palo Alto, California, USA

${ }^{6}$ University of Texas at Houston- Memorial Hermann Center

for Healthcare Quality and Safety, University of Texas Medical

School, Houston, Texas, USA

${ }^{7}$ Perinatal Epidemiology and Health Outcomes Research Unit, Division of Neonatal and Developmental Medicine, Stanford

University School of Medicine and Lucile Packard Children's

Hospital, Palo Alto, California, USA

${ }^{8}$ Levine Cancer Institute, Carolinas Health Care System,

Charlotte, North Carolina, USA

${ }^{9}$ Duke University School of Nursing, Durham, North Carolina, USA

${ }^{10}$ Institute of Nursing Science, University of Basel, Basel, Switzerland

Acknowledgements We are grateful to the local leaders and staff of the CPQCC NICUs who contributed their efforts to this study, and to Judy Milne, the patient safety officer of Duke University Hospital, who provided feedback about this manuscript.

Contributors Agree with the manuscript's results and conclusions: PS, EJT, JBG, CN, AA, MAK, RS and JP. Designed the experiments/the study: JBS, JP, PS, JBG, EJT, CN and MAK. Analysed the data: JBS, JP and AA. Wrote the first draft of the paper: JBS, JP, PS, EJT and RS. Assisted with approach and selection of data inputs: JBS, JP, EJT and JBG. Assisted with interpretation of results: PS, RS, JBS, JP and EJT. Contributed to revision of the paper: JP, JBG, JBS, RN, RS, CN, AA, MAK, PS and EJT.

Competing interests JP's contribution is supported in part by the Eunice Kennedy Shriver National Institute of Child Health and Human Development \#1 K23 HD056298-01 (PI: Profit).

$\mathrm{AA}$ and MAK also receive support from a Veterans

Administration Center Grant (VA HSR\&D CoE HFP90-20).

EJT's effort is supported in part by grants from the Eunice

Kennedy Shriver National Institute of Child Health and Human

Development \#1 K24 HD053771-01 (PI: Thomas). JBS

received support from an Agency for Healthcare Research and Quality (AHRQ) grant \#1UC1HS014246.

Ethics approval The study was approved by the Institutional Review Boards at Stanford University and Baylor College of Medicine.

Provenance and peer review Not commissioned; externally peer reviewed.

\section{REFERENCES}

1 Sharek PJ, Horbar JD, Mason W, et al. Adverse events in the neonatal intensive care unit: development, testing, and findings of an NICU-focused trigger tool to identify harm in North American NICUs. Pediatrics 2006;118:1332-40. 
2 Simpson KR. Measuring perinatal patient safety: review of current methods. J Obstet Gynecol Neonatal Nurs 2006;35:432-42.

3 Frankel A, Graydon-Baker E, Neppl C, et al. Patient safety leadership WalkRounds. Jt Comm J Qual Saf 2003;29: 16-26.

4 Frankel A, Gandhi TK, Bates DW. Improving patient safety across a large integrated health care delivery system. Int J Qual Health Care 2003;15(Suppl 1):i31-40.

5 Zimmerman R, Ip I, Daniels C, et al. An evaluation of patient safety leadership walkarounds. Healthcare Q 2008;11(3 Spec No.):16-20.

6 Budrevics G, O’Neill C. Changing a culture with patient safety walkarounds. Healthcare Quarterly, 8(Sp) October 2005:2025. doi:10.12927/hcq..17657

7 Campbell DA Jr, Thompson M. Patient safety rounds: description of an inexpensive but important strategy to improve the safety culture. Am J Med Qual 2007;22:26-33.

8 Feitelberg SP. Patient safety executive walkarounds. Perm J 2006;10:29-36.

9 Verschoor KN, Taylor A, Northway TL, et al. Creating a safety culture at the Children's and Women's Health Centre of British Columbia. J Pediatr Nurs 2007;22:81-6.

10 Graham S, Brookey J, Steadman C. Patient safety executive Walkarounds. In: Henriksen K, Battles JB, Marks ES, et al., eds. Advances in patient safety: from research to implementation. Vol 4: programs, tools, and products. Rockville, MD: Agency for Healthcare Research and Quality, 2005:223-34.

11 Frankel A, Grillo SP, Pittman M, et al. Revealing and resolving patient safety defects: the impact of leadership WalkRounds on frontline caregiver assessments of patient safety. Health Serv Res 2008;43:2050-66.

12 Thomas EJ, Sexton JB, Neilands TB, et al. The effect of executive walk rounds on nurse safety climate attitudes: a randomized trial of clinical units. BMC Health Serv Res 2005;5:28.

13 Sexton JB, Berenholtz SM, Goeschel CA, et al. Assessing and improving safety climate in a large cohort of intensive care units. Crit Care Med 2011;39:934-9.

14 Paine LA, Rosenstein BJ, Sexton JB, et al. Assessing and improving safety culture throughout an academic medical centre: a prospective cohort study. Qual Saf Health Care 2010;19:547-54.

15 Schwendimann R, Milne J, Frush K, et al. A closer look at associations between hospital leadership walkrounds and patient safety climate and risk reduction: a cross-sectional study. Am J Med Qual 2013;28:414-21.

16 Shanafelt TD, Boone S, Tan L, et al. Burnout and satisfaction with work-life balance among US physicians relative to the general US population. Arch Intern Med 2012;172:1377-85.

17 Aiken LH, Clarke SP, Sloane DM. Hospital staffing, organization, and quality of care: cross-national findings. Int $J$ Qual Health Care 2002;14:5-13.

18 Bellieni CV, Righetti P, Ciampa R, et al. Assessing burnout among neonatologists. J Matern Fetal Neonatal Med 2012;25:2130-4.

19 Profit J, Sharek PJ, Amspoker AB, et al. Burnout in the NICU setting and its relation to safety culture. BMJ Qual Saf 2014;23:806-13.

20 Gould JB. The role of regional collaboratives: the California Perinatal Quality Care Collaborative model. Clin Perinatol 2010;37:71-86.
21 American Academy of Pediatrics Committee on Fetus And Newborn. Levels of neonatal care. Pediatrics 2012;130:587-97.

22 Profit J, Etchegaray J, Petersen LA, et al. The Safety Attitudes Questionnaire as a tool for benchmarking safety culture in the NICU. Arch Dis Child Fetal Neonatal Ed 2012;97:F127-32.

23 Sexton JB, Helmreich RL, Neilands TB, et al. The Safety Attitudes Questionnaire: psychometric properties, benchmarking data, and emerging research. BMC Health Serv Res 2006;6:44.

24 Schwendimann R, Zimmermann N, Kung K, et al. Variation in safety culture dimensions within and between US and Swiss Hospital Units: an exploratory study. BMJ Qual Saf 2013;22:32-41.

25 AHRQ. Trending: Comparing Results over Time. Hospital Survey on Patient Safety Culture: 2010 User Comparative Database Report. Rockville, MD: Agency for Healthcare Research and Quality, 2010.

26 Maslach C, Jackson SE. Maslach Burnout Inventory. Palo Alto, CA: Consulting Psychologists Press, Inc., 1981.

27 Blegen MA, Gearhart S, O’Brien R, et al. AHRQ’s hospital survey on patient safety culture: psychometric analyses. J Patient Saf 2009;5:139-44.

28 Sexton JB, Holzmueller CG, Pronovost PJ, et al. Variation in caregiver perceptions of teamwork climate in labor and delivery units. J Perinatol 2006;26:463-70.

29 Kho ME, Carbone JM, Lucas J, et al. Safety Climate Survey: reliability of results from a multicenter ICU survey. QSHC $2005 ; 14: 273-8$.

30 Modak I, Sexton JB, Lux TR, et al. Measuring safety culture in the ambulatory setting: the safety attitudes questionnaireambulatory version. J Gen Intern Med 2007;22:1-5.

31 Daugherty EL, Paine LA, Maragakis LL, et al. Safety culture and hand hygiene: linking attitudes to behavior. Infect Control Hosp Epidemiol 2012;33:1280-2.

32 Block M, Ehrenworth JF, Cuce VM, et al. Measuring handoff quality in labor and delivery: development, validation, and application of the Coordination of Handoff Effectiveness Questionnaire (CHEQ). Jt Comm J Qual Patient Saf 2013;39:213-20.

33 Sexton JB, Thomas EJ, Pronovost P. The context of care and the patient care team. In: Proctor PR, Compton WD, Grossman HJ, Fanjiang G, eds. Building a better delivery system: a new engineering/health care partnership. Washington, DC: National Academies Press, 2005:119-24.

34 Profit J, Etchegaray J, Petersen LA, et al. Neonatal intensive care unit safety culture varies widely. Arch Dis Child Fetal Neonatal Ed 2012;97:F120-6.

35 Ahola K, Vaananen A, Koskinen A, et al. Burnout as a predictor of all-cause mortality among industrial employees: a 10-year prospective register-linkage study. J Psychosom Res 2010;69:51-7.

36 Fahrenkopf AM, Sectish TC, Barger LK, et al. Rates of medication errors among depressed and burnt out residents: prospective cohort study. BMJ 2008;336:488-91.

37 Kitaoka-Higashiguchi K, Morikawa Y, Miura K, et al. Burnout and risk factors for arteriosclerotic disease: follow-up study. J Occup Health 2009;51:123-31.

38 Mealer M, Burnham EL, Goode CJ, et al. The prevalence and impact of post traumatic stress disorder and burnout syndrome in nurses. Depress Anxiety 2009;26:1118-26.

39 Prins JT, van der Heijden FM, Hoekstra-Weebers JE, et al. Burnout, engagement and resident physicians' self-reported errors. Psychol Health Med 2009;14:654-66. 
40 Shanafelt TD, Balch CM, Bechamps G, et al. Burnout and medical errors among American surgeons. Ann Surg 2010;251:995-1000.

41 Shanafelt TD, Bradley KA, Wipf JE, et al. Burnout and self-reported patient care in an internal medicine residency program. Ann Intern Med 2002;136:358-67.
42 West CP, Huschka MM, Novotny PJ, et al. Association of perceived medical errors with resident distress and empathy: a prospective longitudinal study. JAMA 2006;296:1071-8.

43 Seligman ME, Steen TA, Park N, et al. Positive psychology progress: empirical validation of interventions. Am Psychol 2005;60:410-21. 